\title{
SIGNO DEL YIN-YANG: LA DUALIDAD DEL PSEUDOANEURISMA
}

Drs. Italo Cavallo $B^{(1)}$, Cristian Quezada $J^{(1)}$, Lionel Suazo $R^{(2)}$.

1. Médico Becado Imagenología Hospital Clínico Universidad de Chile, Santiago-Chile.

2. Médico Radiólogo Hospital Clínico Universidad de Chile, Santiago-Chile.

\section{YIN -YANG SIGN: THE DUALITY OF THE PSEUDOANEURYSM}

\begin{abstract}
Pseudoaneurysm is a collection of blood adjacent to a punctured blood vessel, connected with it and characterized by an inner bidirectional flow in relation with systole and diastole.

This diagnosis is usually based on color Doppler examination findings, among which we can highlight the visualization of a collection with a two-way flow pattern, with a red and a blue component, which resembles the Chinese Yin Yang symbol. The increase in the number of angiographic procedures and arterial catheterization requires from radiologists the ability to properly diagnose the complications these procedures may entail.

Keywords: Color Doppler ultrasound, Pseudoaneurysm, Yin-Yang Sign.
\end{abstract}

Resumen:El pseudoaneurisma corresponde a una colección adyacente a un vaso arterial puncionado, conectada con éste y con flujo bidireccional en su interior, en relación con el sístole y el diástole. Habitualmente este diagnóstico se basa en los hallazgos al estudio Doppler color, entre los cuales destaca la visualización de una colección que presenta flujo en dos direcciones, que se representa en dos colores y se asemeja al signo oriental del Yin Yang. El aumento en el número de procedimientos angiográficos y cateterizaciones arteriales requiere que los radiólogos diagnostiquen adecuadamente las complicaciones que estos procedimientos pueden presentar.

Palabras clave: Ecografía Doppler color, Pseudoaneurisma, Signo del Yin-Yang.

Cavallo I y cols. Signo del Yin-Yang: La dualidad del pseudoaneurisma. Rev Chil Radiol 2010; 16(1): 36-38.

Correspondencia: Dr. Italo Cavallo $B$.

italocavallo@gmail.com

Trabajo recibido el 24 de septiembre de 2009, aceptado para publicación el 26 de enero 2010.

\section{Aspecto imaginológico}

En ultrasonido, el signo del Yin-Yang (Figura 1) corresponde al flujo sanguíneo de entrada y salida observado en un pseudoaneurisma al estudio Doppler color, que se asemeja a la imagen del signo chino ancestral que representa la dualidad de todos los elementos existentes en el universo.

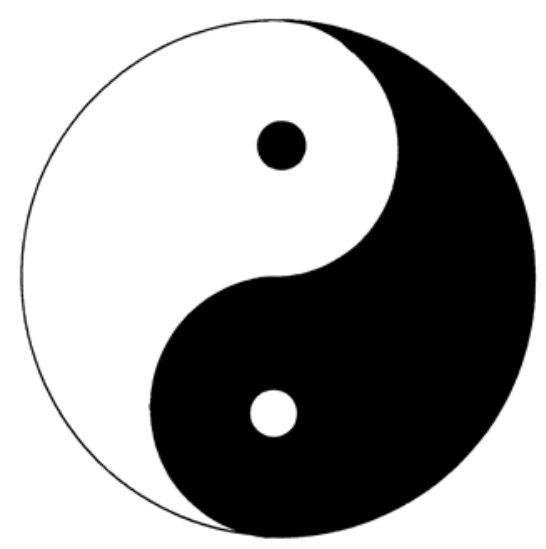

Figura 1. Signo chino del Yin-Yang, que representa la dualidad de los elementos del universo.

En el caso de los pseudoaneurismas post punción, se produce una lesión de la pared vascular y se forma una colección extraluminal de sangre conectada con el vaso principal, que es contenida por el hematoma y los tejidos blandos adyacentes ${ }^{(1)}$ (Figura 2). Clínicamente se presenta como un aumento de volumen pulsátil asociado a equimosis, que puede ir aumentando de tamaño, en relación al sitio de punción.

Ecográficamente, esta lesión aparece como una imagen redondeada hipoecogénica, conectada al vaso lesionado a través de un cuello que muestra un flujo arremolinado de distintos colores al estudio Doppler color, que representa el flujo anterógrado durante el sístole y al flujo retrógrado durante el diástole, en la porción no trombosada del pseudoaneurisma ${ }^{(2,3)}(\mathrm{Fi}$ gura 2). Característicamente, el estudio con Doppler espectral muestra una curva con flujo anterógrado y 

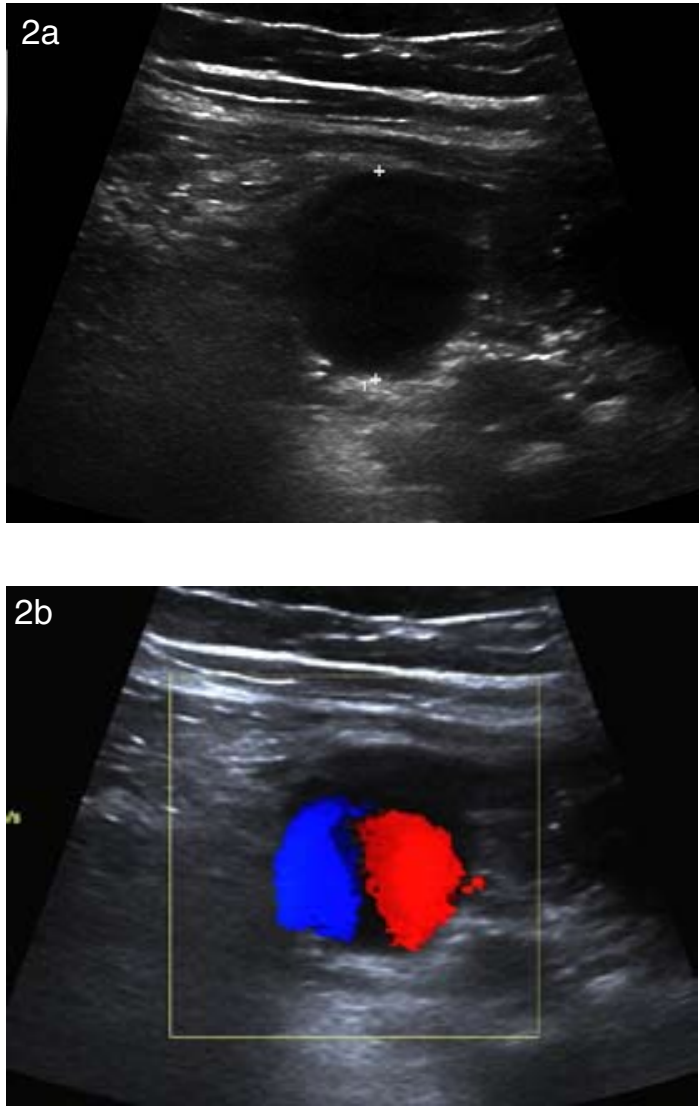

Figura 2 a,b. Se observa una imagen hipoecogénica, redondeada, que al examen Doppler color presenta flujo anterógrado y retrógrado, signo de Yin Yang.

retrógrado en el cuello del pseudoaneurisma, lo que en la literatura anglosajona se conoce como "to-andfro" (2) (Figura 3).

El angio-TAC también permite diagnosticar y caracterizar los pseudoaneurismas, si bien no se utiliza en la práctica habitual. El uso de la angiografía convencional, se restringe a los pacientes que requieren terapia intervencional.

\section{Diagnóstico diferencial}

En el contexto de un paciente sometido a un procedimiento o punción vascular, la imagen hipoecogénica al estudio ecográfico en escala de grises puede plantear el diagnóstico diferencial de hematoma o colección adyacente al sitio de punción ${ }^{(2)}$. El estudio dirigido con Doppler color demuestra una conexión entre esta imagen y una arteria adyacente, lo que sumado a la presencia de flujo anterógrado y retrógrado con el ciclo cardíaco, excluye cualquier posibilidad diferente de pseudoaneurisma.

\section{Discusión}

Los pseudoaneurismas corresponden a una complicación poco frecuente de una punción arterial,
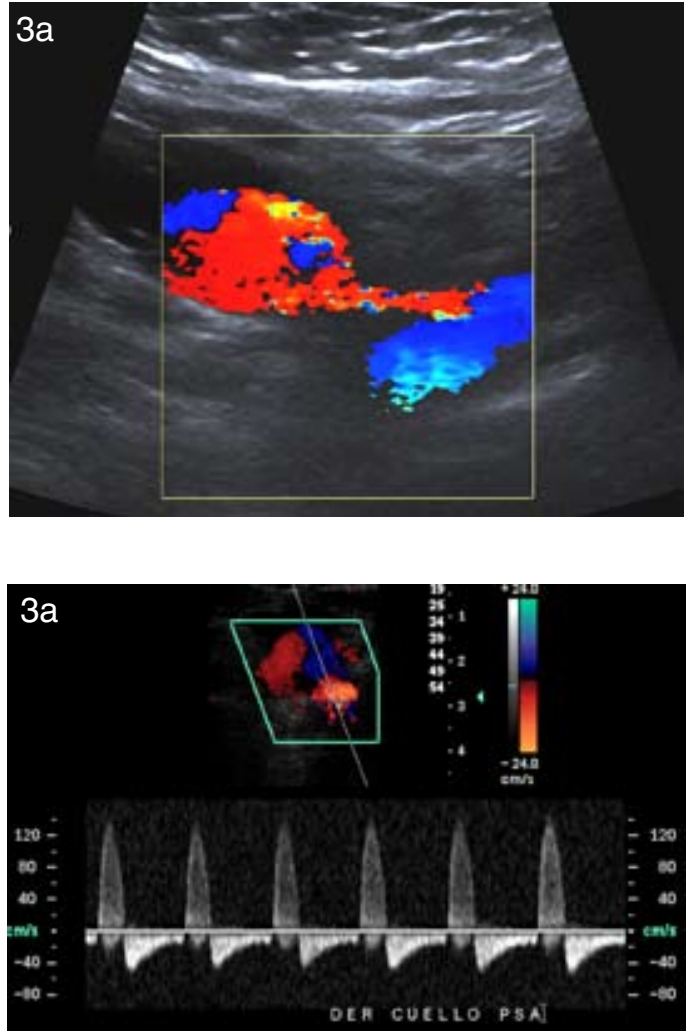

Figura 3 a,b. El Doppler muestra la relación entre el pseudaneurisma y el vaso adyacente, conectados a través de un fino cuello. El Doppler espectral presenta la curva característica "to and fro".

típicamente observada luego de la cateterización para procedimientos angiográficos diagnósticos 0 terapéuticos; también pueden desarrollarse en relación a traumatismos.

Su incidencia ha ido en aumento en la medida en que ha crecido la realización de procedimientos intervencionales. Se han descrito incidencias de $0.2 \mathrm{a}$ $0.5 \%$, luego de angiografías diagnósticas y de hasta 2 a $8 \%$, después de angioplastías coronarias, según algunas series ${ }^{(2)}$.

La arteria femoral común corresponde a la vía de acceso más utilizada en procedimientos angiográficos, por lo cual, es el lugar donde más frecuentemente es posible observar pseudoaneurismas, aunque éstos pueden desarrollarse en cualquier vaso arterial.

El manejo de los pseudoaneurismas post-punción puede realizarse de 3 formas:

- No invasiva, que consiste en la compresión del cuello del pseudoaneurisma guiada por ecografía. De este modo se colapsa el lumen del trayecto que une el pseudoaneurisma con el vaso adyacente, produciendo una trombosis a este nivel. La tasa de éxito de este tratamiento va de $66 \%-88 \%$ según la serie ${ }^{(4)}$. 
- Mediante la inyección de trombina en el pseudoaneurisma, con la finalidad de trombosar la cavidad.

- Instalando coils en la cavidad del pseudoaneurisma o stent en el trayecto que comunica el pseudoaneurisma con la arteria, con la finalidad de obstruir el flujo y provocar una trombosis local.

\section{Conclusión}

El signo del Yin-Yang se observa en el interior de las colecciones adyacentes a vasos arteriales; se origina en las diferentes direcciones del flujo en la cavidad, que le otorga un aspecto arremolinado con un componente rojo y otro azul, que asemeja la forma del signo chino de la dualidad del universo. El estudio espectral demuestra flujo bidireccional y evidencia la conexión del pseudoaneurisma con el vaso arterial.

\section{Bibliografía}

1. Krebs C, Giyanani V, Eisenberg R. Pseudoaneurisma. Doppler Color. Marbán. 2001; p. 421-425.

2. Alan Dubbins. Clinical Doppler Ultrasound. Harcourt Publishers Limited 2000; 73-101.

3. Lupatelli T. The Yin Yang Sign. Radiology 2006; 238: 1070-1071.

4. Morgan R. Current Treatment Methods for Postcatheterization Pseudoaneurysms. J Vasc Interv Radiol 2003; 14: 697-710. 